\title{
ISOMORPHISMS OF GROUP ALGEBRAS
}

\author{
ROBERT S. STRICHARTZ ${ }^{1}$
}

1. Introduction. In [1] Edwards considers bipositive isomorphisms of $L^{p}$ convolution algebras of compact groups, $1 \leqq p<\infty$, and shows they are all induced by bicontinuous isomorphisms of the groups. He asks if a similar result holds if we assume the algebra isomorphism is isometric instead of bipositive. If $p=2$ the result is false. If $p \neq 2$ we will establish the result, including the case $p=\infty$.

For $p \neq 2$, we will show that right translation operators on $L^{p}(G)$ can be characterized (ignoring constants of absolute value 1) as isometric, invertible, left multipliers. Since these properties are preserved by an isometric isomorphism we will obtain an isomorphism of the underlying groups. To handle the case $p=\infty$ we will show that an isomorphism $L^{\infty}\left(G_{1}\right) \rightarrow L^{\infty}\left(G_{2}\right)$ maps $C\left(G_{1}\right) \rightarrow C\left(G_{2}\right)$ and then apply the results of $[1]$.

2. Multipliers. Let $G$ be a locally compact group with right Haar measure $d m$. All our results deal with $L^{p}(G, d m)=L^{p}(G)$, either real or complex. If $y \in G$ then let $\rho_{y} f(x)=f\left(x y^{-1}\right)$. A (left) multiplier on $L^{p}(G), 1 \leqq p<\infty$, is a bounded linear operator $R$ on $L^{p}(G)$ which commutes with every $\rho_{y}$.

We define the convolution of two $L^{1}$ functions by the formula $f * g(x)=\int_{G} f\left(x y^{-1}\right) g(y) d m(y)=\int_{G} \rho_{y} f(x) g(y) d m(y) . \quad L^{1}(G)$ forms a Banach algebra under this multiplication. If $G$ is compact, and we assume the Haar measure is normalized so that $m(G)=1$, then $L^{p}(G)$ forms a Banach algebra under convolution for $1 \leqq p \leqq \infty$.

Proposition. If $G$ is compact, or $p=1$, and $R$ is a bounded linear operator on $L^{p}(G)$, then $R$ is a multiplier if and only if

$$
R(f * g)=(R f) * g, \quad \text { for every } f, g \in L^{p}(G) .
$$

Proof. Suppose (1) holds. Let $C_{a}$ be any compact neighborhood of $y \in G$, and $g_{a}=\left(1 / m\left(C_{a}\right)\right) \phi_{a}$, where $\phi_{c}$ is the characteristic function of $C_{a}$. Then for any $f \in L^{p}$, as $C$ converges to $y, R\left(f * g_{a}\right) \rightarrow R \rho_{y} f$ and $(R f) * g_{a} \rightarrow \rho_{y} R f$. Thus $R$ is a multiplier.

Conversely, if $R$ is a multiplier and $f, g$ are step functions, then

Received by the editors December 6, 1965.

1 This work was done while the author held a National Science Foundation Fellowship. 


$$
\begin{aligned}
R(f * g) & =R\left(\int_{G} \rho_{y} f(x) g(x) d m(x)\right) \\
& =\int_{G} R \rho_{y} f(x) g(x) d m(x) \\
& =\int_{G} \rho_{y} R f(x) g(x) d m(x) \\
& =(R f) * g .
\end{aligned}
$$

The identity holds for arbitrary $L^{p}$ functions by continuity.

Our main tool is a theorem of Banach and Lamperti [2]. We state it in a form slightly different from that of [2], but virtually no modification of the proof given there is necessary: ${ }^{2}$

THEOREM. If $(X, \Sigma, m)$ is a measure space and $R: L^{p}(X) \rightarrow L^{p}(X)$ ' $p \neq 2, \infty$, is an isometry, then there is a $\sigma$-ring isomorphism $T$ of $\Sigma^{\prime}$ into itself, where $\Sigma^{\prime}$ is the $\sigma$-ring generated by the sets of finite measure modulo the sets of measure zero, and a measurable function $h$ on $X$, such that

$$
R \phi_{E}(x)=h(x) \phi_{T E}(x) \text { a.e., }
$$

for $\phi_{E}$ the characteristic function of a set $E$ of finite measure. If $R$ is invertible then $T$ is.

Theorem 1. Let $G$ be a locally compact group, and let $R$ be a left multiplier and $L^{p}(G), p \neq 2$, which is an invertible isometry. Then

$$
R f(x)=c f\left(x y^{-1}\right)=c \rho_{y} f(x),
$$

for some $y \in G$ and constant $c,|c|=1$.

Proof. Let $y \in G$ be fixed. We will show $\rho_{y} h=h$, where $h$ is the function in (2). For if not there exists a set $E$ of finite positive measure such that $h\left(x y^{-1}\right) \neq h(x)$ for $x \in E$. Now the sets $T^{-1} E$ and $T^{-1}\left(E y^{-1}\right)$ may have $\sigma$-finite measure; but by taking a suitable subset of $E$, still of positive measure, we can assure that they have finite measure. Having done this, let $F=T^{-1}\left(E y^{-1}\right) \cup\left(T^{-1} E\right) y^{-1}$. Then for $x \in E$ we have on the one hand $\rho_{y} R \phi_{F}(x)=R \phi_{F}\left(x y^{-1}\right)=h\left(x y^{-1}\right) \phi_{T F}\left(x y^{-1}\right)$ $=h\left(x y^{-1}\right)$ since $x y^{-1} \in E y^{-1} \subseteq T F$, and on the other hand $R \rho_{y} \phi_{F}(x)$ $=R \phi_{F y}(x)=h(x) \phi_{T(F y)}(x)=h(x)$ since $E \subseteq T(F y)$. Thus $h(x)=h\left(x y^{-1}\right)$ for almost every $x \in E$, a contradiction.

Since $h$ is invariant under translation it must be a constant, say $k$.

2 The assumption in [2] that $m$ is $\sigma$-finite is not needed for our weaker conclusion because the support of every $L^{p}$ function is $\sigma$-finite. 
It follows easily from (2) that $R$ is now a bounded operator on every $L^{p}$ class, in particular $L^{1}$, and hence must be convolution with a finite measure. It also follows from (2) that $\bar{k}$ times this measure is positive and invertible which implies that it must be a constant times a point mass. Since $R$ is an isometry the constant must have absolute value one.

3. Isomorphisms of $L^{p}, p \neq 2, \infty$. We can now prove our main Theorem. For $p=1$ it was first proved by Wendel [4]. See also [5] for a strengthened version.

Theorem 2. Let $U: L^{p}\left(G_{1}\right) \rightarrow L^{p}\left(G_{2}\right), p \neq 2, \infty, G_{1}$ and $G_{2}$ compact unless $p=1$, be an isometric algebra isomorphism. Then there exists a bicontinuous group isomorphism $A: G_{2} \rightarrow G_{1}$ and a character $\chi$ on $G_{2}$ (a continuous homomorphism to the circle group, real-valued in the case of real $\left.L^{p}\right)$ such that

$$
U f(x)=J_{\chi}(x) f(A x),
$$

for all $f \in L^{p}\left(G_{1}\right)$, where $J$ is a constant equal to the Jacobian of $A^{-1}$, which must be 1 if $p \neq 1$.

Proof. We note that each group is anti-isomorphic with its right translation operators, and the topology of the group coincides with the strong operator topology on the translation operators. Given $y \in G_{2}$, the operator $U^{-1} \rho_{y} U$ on $L^{p}\left(G_{1}\right)$ is an invertible isometric multiplier, hence $U^{-1} \rho_{y} U=c \rho_{z}$ for some constant $c,|c|=1$ and $z \in G_{1}$. Define $A y=z$ and $\chi(y)=c^{-1}$. We see from $\chi(x y)^{-1} \rho_{A(x y)}=U^{-1} \rho_{x y} U$ $=U^{-1} \rho_{y} U U^{-1} \rho_{x} U=\chi(x)^{-1} \chi(y)^{-1} \rho_{A y} \rho_{A x}=\chi(x)^{-1} \chi(y)^{-1} \rho_{A x A y}$ that $\chi$ is a character on $G_{2}$ and $A$ is a group homomorphism. Since $U$ is invertible $A$ is an isomorphism. Regarded as an isomorphism of translation operators $A$ is bicontinuous in the strong operator topology. Thus $A$ is a bicontinuous isomorphism of $G_{2}$ on to $G_{1}$.

It remains to verify (4). We first note that if we define $T f(x)$ $=J \chi(x) f(A x)$ then $T$ is an isometric isomorphism and $T^{-1} \rho_{y} T$ $=\chi(y)^{-1} \rho_{A y}$. These are straightforward computations. It follows that $U T^{-1}$ is an isometric isomorphism and a multiplier. By Theorem 1 it must have the form (3). But it is easy to see that the only operator of the form (3) which is an algebra isomorphism is the identity. Thus $U=T$; i.e. $U$ is of the form (4).

4. Isomorphisms of $L^{2}$ and $L^{\infty}$. To see why Theorem 2 is false for $L^{2}$ consider a compact abelian group $G$. By the Plancherel Theorem the convolution algebra $L^{2}(G)$ is isometrically isomorphic to the 
pointwise multiplication algebra $L^{2}(\hat{G}), \hat{G}$ the dual group of $G$. Thus the algebra $L^{2}(G)$ is completely determined by the cardinality of $\hat{G}$. For a discussion of the nonabelian case see [3, Chapter 8]. It follows from the results there that $L^{2}\left(G_{1}\right)$ and $L^{2}\left(G_{2}\right)$ are isometrically isomorphic if and only if for every positive integer $k$, the cardinality of the set of equivalence classes of irreducible representations of dimension $k$ of $G_{1}$ is equal to the cardinality of the corresponding set for $G_{2}$.

To reduce the $L^{\infty}$ case to the results of [1] we first prove:

LEMma. If $G_{1}$ and $G_{2}$ are compact groups, and $T: L^{\infty}\left(G_{1}\right) \rightarrow L^{\infty}\left(G_{2}\right)$ is a continuous algebra isomorphism then $T$ maps $C\left(G_{1}\right)$, the continuous functions on $G_{1}$, onto $C\left(G_{2}\right)$.

Proof. Consider first the complex algebras. $T$ must send minimal two-sided ideals of $L^{\infty}\left(G_{1}\right)$ to minimal two-sided ideals of $L^{\infty}\left(G_{2}\right)$. Now to every irreducible representation of $G_{1}$ is associated a minimal twosided ideal generated by its entry functions. By the Peter-Weyl Theorem these ideals span a dense subspace of $C\left(G_{1}\right)$. Furthermore, there are no other minimal two-sided ideals. For if $I$ were any other such ideal, $f \in I$, and $g$ an element of one of the above ideals, then $f * g=0$, and hence the same equation holds for all $g \in C\left(G_{1}\right)$ by continuity. But this implies $f=0$, hence $I$ is the trivial ideal. Thus $T$ maps a dense subspace of $C\left(G_{1}\right)$ into $C\left(G_{2}\right)$, hence all of $C\left(G_{1}\right)$ by continuity. The same argument applied to $T^{-1}$ shows that $C\left(G_{2}\right)$ actually is mapped onto $C\left(G_{2}\right)$.

If we are dealing with the real $L^{\infty}$ algebras we must first extend $T$ to the complex $L^{\infty}$ by defining $T(f+i g)=T f+i T g$. Then $T$ satisfies the hypotheses for complex $L^{\infty}$, hence the conclusion holds for complex continuous functions. But since $T$ maps real functions to real functions, the conclusion also holds for real continuous functions.

THEOREM 3. Let $G_{1}$ and $G_{2}$ be compact groups (with normalized Haar measure), and let $U: L^{\infty}\left(G_{1}\right) \rightarrow L^{\infty}\left(G_{2}\right)$ be an algebra isomorphism which is either (a) isometric, or (b) bipositive. Then there exists a bicontinuous group isomorphism $A: G_{2} \rightarrow G_{1}$ and a character $\chi$ (in case (b) $\chi=1$ ) on $G_{2}$ such that

$$
U f(x)=\chi(x) f(A x) \quad \text { for all } f \in L^{\infty}\left(G_{1}\right) .
$$

Proof. By the Lemma, $U$ maps $C\left(G_{1}\right)$ onto $C\left(G_{2}\right)$. By [1, Theorem 2] $U$ has the form (5) for all $f \in C\left(G_{1}\right)$. To show that (5) holds for all $g \in L^{\infty}\left(G_{1}\right)$ we use the fact that $C\left(G_{1}\right)$ is an ideal in $L^{\infty}\left(G_{1}\right)$. Let $f \in C\left(G_{1}\right)$. Then $f * g \in C\left(G_{1}\right)$ hence 


$$
\begin{aligned}
U(f * g)(x) & =\chi(x) \int_{G_{1}} f\left((A x) t^{-1}\right) g(t) d t \\
& =\chi(x) \int_{G_{2}} f\left(A\left(x y^{-1}\right)\right) g(A y) d y \\
& =U f * U g(x)=\int_{G_{2}} f\left(A\left(x y^{-1}\right)\right) \chi(x) \chi\left(y^{-1}\right) U g(y) d y .
\end{aligned}
$$

Since this holds for all $f \in C\left(G_{1}\right)$, it follows that $\chi\left(y^{-1}\right) U g(y)=g(A y)$, which is (5).

\section{BIBLIOGRAPHY}

1. R. E. Edwards, Bipositive and isometric isomorphisms of some convolution algebras, Canad. J. Math. 17 (1965), 839-846.

2. J. Lamperti, On the isometries of certain function spaces, Pacific J. Math. 8 (1958), 459-466.

3. L. Lommis, An introduction to abstract harmonic analysis, Van Nostrand, New York, 1953.

4. J. G. Wendel, On isometric isomorphism of group algebras, Pacific J. Math. 1 (1951), 305-311.

5. - Left centralizers and isomorphisms of group algebras, Pacific J. Math. 2 (1952), 251-261.

Princeton University 